\title{
DESAFIOS DA INDÚSTRIA FARMACÊUTICA BRASILEIRA
}

\author{
Angelo C. Pinto* e Eliezer J. Barreiro \\ Instituto Nacional de Ciência e Tecnologia de Fármacos e Medicamentos (INCT-INOFAR), Av. Carlos Chagas Filho, 373, Centro de Ciências \\ da Saúde (CCS), Bloco K, $2^{\circ}$ andar, Sala 23, Cidade Universitária; CP 68043, 21944-971 Rio de Janeiro - RJ, Brasil
}

Recebido em 15/10/13; aceito em 1/11/13; publicado na web em 4/11/13

\begin{abstract}
CHALLENGES OF THE BRAZILIAN PHARMACEUTICAL INDUSTRY. The paper traces a panorama of the development of new drugs and hopes to contribute for Brazil to become a player in the discovery of new drugs. Brazil is the sixth world market retail consumer of medicines prone to expansion, has a pharmaceutical industry focused on the production of generics and a very large number of undergraduate courses in Pharmacy. The national industry has grown over the last decade after the Generics Act 9787/99. Despite these positive aspects, a number of bottlenecks prevent Brazilian pharmaceutical industry to invest in the development of new drugs. There are, however, a number of initiatives to reduce the dependence on imported generic drugs. It is a very good start for the development of new pharmaceutical drugs.
\end{abstract}

Keywords: Brazilian pharmaceutical industry; generic medicines; bottlenecks of the Brazilian pharmaceutical industry.

\section{INTRODUÇÃo}

A Diretoria e o Conselho Consultivo da Sociedade Brasileira de Química, atenta aos desafios contemporâneos, agravados pela globalização, com o objetivo de contribuir para a formulação de políticas de Ciência e Tecnologia (C\&T) no domínio da Química, vem elaborando propostas e documentos, entre os quais se destacam os Eixos Mobilizadores em Química ${ }^{1}$ e Química sem Fronteiras, ${ }^{2}$ para melhorar, em todos os níveis, a formação do profissional químico e aumentar a competitividade do país no setor Químico.

O setor químico-farmacêutico representa um dos maiores desafios do país e estes só poderão ser enfrentados se empresários, governo e academia estiverem juntos.

$\mathrm{Na}$ área farmacêutica, mesmo com o fortalecimento das farmacoquímicas devido à lei que estabeleceu o comércio de fármacos genéricos, as empresas limitam-se, em sua grande parte, a formular e embalar os princípios ativos que importam principalmente da Índia e da China, re-editando, em versão contemporânea, o "Caminho das Índias". Estas importações aumentam em muito o déficit na balança comercial brasileira. O governo brasileiro, ao formular as Parcerias Público-Privadas (PPPs), deu um passo em direção ao fortalecimento do setor farmacêutico.

Entre os setores industriais, a cadeia farmacêutica é uma das mais inovadoras, daí a necessidade dos altos investimentos em pesquisa e desenvolvimento para a busca de novos fármacos. Em contrapartida, o setor farmacêutico é um dos mais rentáveis em escala global, e, por isso, é dos mais competitivos. Esta competição por aumento da rentabilidade vem levando as Big Pharmas a sucessivas fusões ou a adquirirem empresas menores, como se observou em tempos recentes. No Brasil, nos últimos anos, do mesmo modo que aconteceu em escala global, ${ }^{3}$ ocorreram fusões entre empresas brasileiras, aquisição de empresas farmacêuticas brasileiras por empresas estrangeiras e de nacionais por nacionais. ${ }^{3}$ Por exemplo, o Aché laboratório Farmacêutico S. A. adquiriu o laboratório Biosintética Farmacêutica Ltda. ${ }^{4}$

Outra das características do setor farmacêutico é a qualificação acadêmica dos pesquisadores em funções de P \& D em síntese orgânica, e a expertise de seus profissionais químicos e engenheiros químicos em adaptarem as etapas de síntese de bancada para

*e-mail: angelocpinto@gmail.com.br o escalonamento primário, indispensável para qualquer tecnologia de síntese multietapas em maior escala. A necessidade contínua em inovar do setor industrial farmacêutico impõe ambiente competitivo que demanda alta capacitação profissional, razão pela qual as Big Pharmas têm elevado número de doutores atuando em seus quadros de $\mathrm{P} \& \mathrm{D}$, sendo parte significativa de sua força de trabalho. ${ }^{5}$ Por outro lado, poucas são as empresas farmacêuticas brasileiras que têm pessoal deste nível de qualificação entre seus colaboradores. Mesmo essas quando os têm é em número reduzido em relação ao total de colaboradores contratados.

A história da indústria farmacêutica brasileira é repleta de altos e baixos, e de uma forma ou de outra, sempre esteve vinculada ao estado. O melhor exemplo disso é o caso da produção de soros e vacinas, no início do século XX, em Manguinhos (RJ) e no Instituto Bacteriológico de São Paulo. ${ }^{6}$

A indústria farmacêutica moderna surgiu após a segunda guerra mundial, com a entrada da penicilina na terapêutica médica e com as grandes corporações farmacêuticas. ${ }^{7}$ Como foge ao escopo deste artigo e há fontes primárias que tratam do tema, não abordaremos a história da indústria farmacêutica no Brasil. ${ }^{8}$

\section{A INOVAÇÃO EM FÁRMACOS}

Levantamento da Thomson Reuters mostra que o número de fármacos inovadores ${ }^{9}$ nos últimos 10 anos vem caindo progressivamente. ${ }^{10,11}$ Em 2010, 21 novas entidades químicas foram

aprovadas pela agência regulatória norte-americana, 4 a menos do que em 2009. O menor número de inovações ocorreu em 2004 (Figura 1). ${ }^{11}$ De fato, os primeiros anos da década não foram muito alvissareiros para as Big-Pharmas, que viram despencar de 35, em 1999 e 2000, para 19 novos fármacos, no ano de 2004. Esta situação foi objeto de estudo por vários e numerosos especialistas e tema de muitos artigos publicados em prestigiosas revistas científicas. Várias foram as possíveis causas desta crise de inovação que caracterizou o início do século.

Apesar de o aumento dos investimentos em PD\&I no setor, para alguns autores, ser $\mathrm{ca} .10-12 \%$ do faturamento bruto dessas empresas, ${ }^{12}$ diminuiu o número de novas pequenas moléculas de fármacos lançadas no mercado. Esta situação provocou mudanças estruturais importantes na gestão da inovação no setor industrial farmacêutico, 
sendo o modelo aberto adotado por várias empresas. Esta nova forma de gestão tecnológica do conhecimento adotada pode explicar a notável aproximação de importantes Big-Pharmas a determinados grupos de pesquisa identificados em universidades, ${ }^{13-15}$ inclusive em algumas brasileiras. Cada vez é maior nas nações desenvolvidas a parceria entre as Big-Pharmas e a academia e vários autores trataram, recentemente, deste tema. ${ }^{16}$

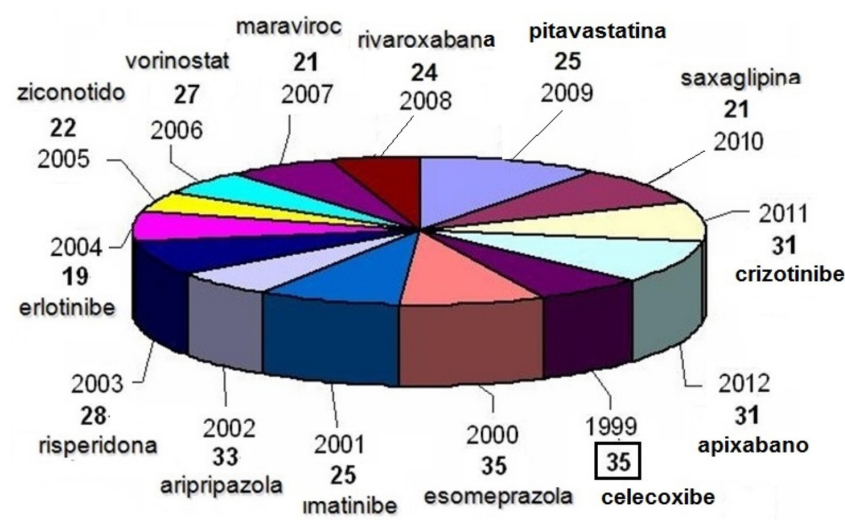

Figura 1. Número de novos fármacos lançados anualmente no período de 1999 até 2012, segundo aprovação da agência regulatória Food Drug Administration dos EUA, e exemplos de fármacos inovadores. (Fonte: Annual Reports in Medicinal Chemistry, Market to Market, Volumes 36-48, Divisão de Química Medicinal, ACS, 1999-2012)

A construção do conhecimento científico, em que se baseiam as modernas inovações terapêuticas, se dá principalmente na academia. Não são raros os exemplos de pesquisadores que foram laureados com o prêmio Nobel, seja de Medicina e Fisiologia ou de Química, ao longo do século 20, desde Paul Ehrlich, que tiveram seus trabalhos de pesquisa relacionados com o surgimento de alguma inovação terapêutica relevante. Pode-se observar também que alguns dos

laureados eram pesquisadores com fortes vínculos ou que atuavam em laboratórios industriais, como exemplifica o caso de Sir James W. Black, Gertude B. Elion e George H. Hitchings, premiados em $1988,{ }^{17}$ responsáveis pela invenção de fármacos inovadores marcantes na terapêutica como propranolol e cimetidina (1), aciclovir (2) e azatioprina (3) (Figura 2), respectivamente.

Quando debruçamo-nos sobre a história da descoberta da sinvastatina (4, Figura 2), lançada em 1986 com o nome fantasia Zoccor ${ }^{\circledR}$, primeiro representante da classe dos antilipêmicos inibidores da enzima hidroximetil-glutarilCo-A redutase (HMGCo-AR) onde se situam as estatinas, a classe de fármacos com o maior faturamento mundial em toda a história dos medicamentos, podemos claramente identificar que, de um lado, a estratégia adotada pela Merck para sua descober$\mathrm{ta}^{18}$ fundamentou-se em conhecimento público baseado em descobertas científicas originadas na Universidade do Texas, em Dallas, EUA. Estas descobertas foram lideradas por Joseph L. Goldstein e Michael S. Brown, que esclareceram o mecanismo de regulação do metabolismo do colesterol e, por isso, receberam em 1985 o prêmio Nobel de Medicina. Esta história praticamente se repete na invenção do imatinibe (5; Figura 2), pela empresa suiça Novartis, em 2001, primeiro fármaco inibidor de tirosina-quinase da classe dos tinibes, que revolucionou o tratamento do câncer. ${ }^{19,20}$ Também neste caso, o estudo das proteínas quinases como possível alvo terapêutico para o tratamento do câncer ${ }^{21}$ foi motivado pela iniciativa acadêmica de Edwin G Krebs e Edmond H Fischer na Universidade de Washington, em Seattle, EUA. Estes cientistas elucidaram o papel desta classe de enzimas e por este notável trabalho científico receberam, em 1992, o prêmio Nobel de Medicina.

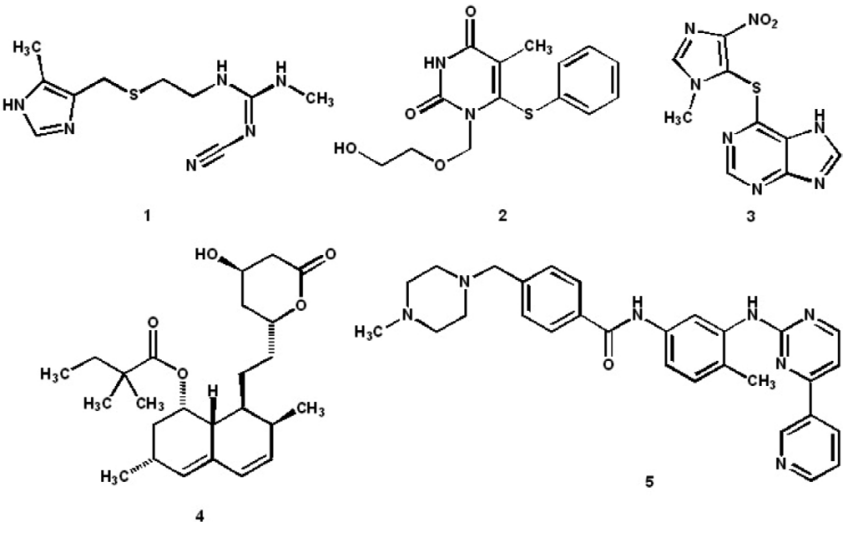

Figura 2. Estruturas dos fármacos cimetidina (1), aciclovir (2), azatiaprina (3), sinvastatina (4) e imatine (5)

\section{MERCADO VAREJISTA BRASILEIRO DE MEDICAMENTOS}

O mercado varejista de medicamentos e o número de instituições de ensino superior de Farmácia no Brasil crescem em ritmo chinês. O Brasil será em 2015 o $6^{0}$ maior mercado consumidor de medicamentos no mundo, sendo estimado por alguns para o corrente ano de 2013 em $c a$. de R 54 bilhões. ${ }^{22}$ Em 2010, estavam registradas 82.204 farmácias e drogarias, 7.351 farmácias de manipulação e 1.053 farmácias homeopáticas no Conselho Federal de Farmácia. O faturamento do setor de farmácias e drogarias atingiu, em 2012, a cifra de 49,6 bilhões de reais e a previsão é que em 2017, a permanecer o atual ritmo de crescimento, chegue a 100 bilhões de reais (Figura 3).

\section{Faturamento do setor comercial de farmácias}

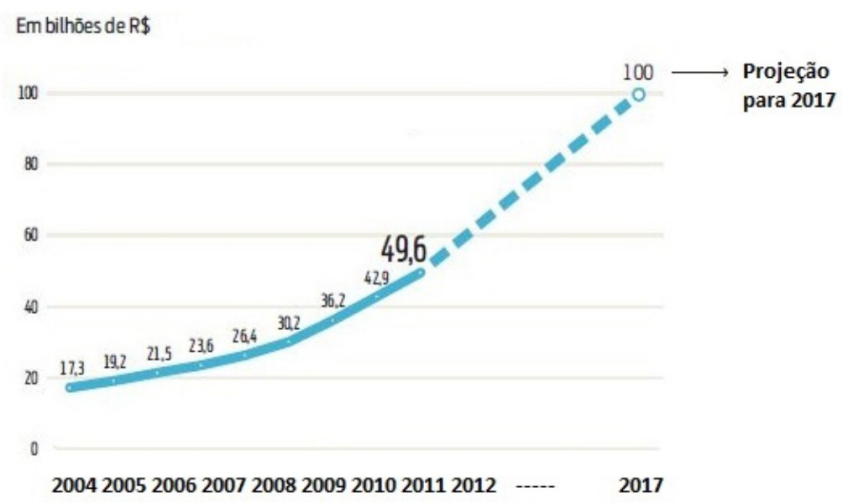

Figura 3. Faturamento do setor comercial de farmácias no Brasil Adaptado de Brasilpar; Infografia: Gazeta do Povo

O mercado de varejo farmacêutico é tão atraente que grandes redes internacionais de farmácias começam a estabelecer-se no país, e fusões de grandes drogarias, como as que ocorreram em 2011, entre Droga Raia e Drogasil e as Drogarias Pacheco e São Paulo, resultaram em grandes gigantes do setor. ${ }^{23} \mathrm{Se}$ for levado em consideração que o consumo per capita anual do brasileiro com medicamentos é baixo, até mesmo quando comparado com o do argentino, e que a renda média da população no Brasil vem aumentando na última década, há muito espaço para mais crescimento no setor (Figura 4).

Outros números que chamam a atenção dos que se dedicam a estudar o setor farmacêutico brasileiro é o de cursos de graduação de Farmácia. Em 2012, estavam cadastrados 481 cursos no Ministério 


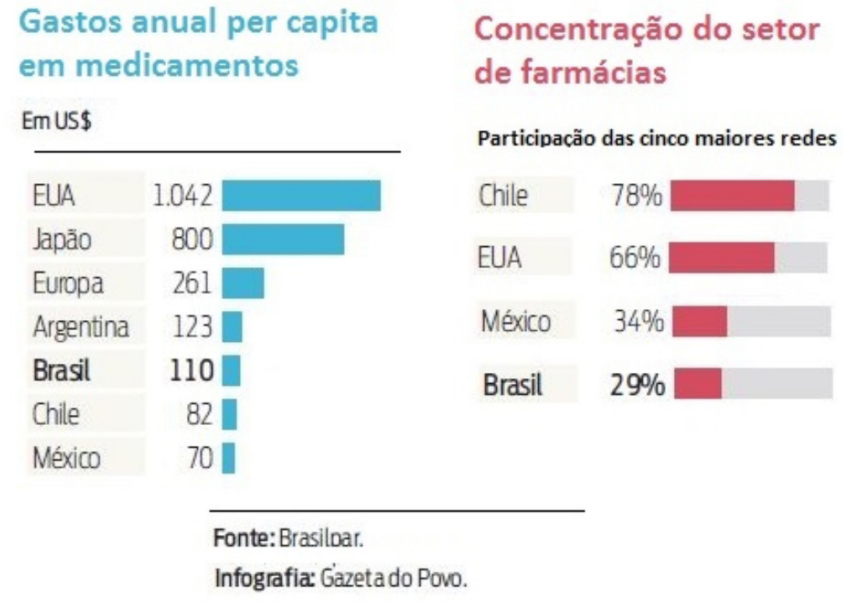

Figura 4. Gastos per capita em medicamentos em alguns países e a situação do mercado varejista farmacêutico no Brasil. (Fonte: Brasilpar)

de Educação. Desses cursos, $83 \%$ (Ca. 400) são de instituições particulares. $^{24}$

\section{OS MEDICAMENTOS GENÉRICOS}

A lei que criou no Brasil os genéricos (n 9787/99) é um divisor de águas na indústria farmacêutica brasileira. Além de facilitar aos brasileiros o acesso aos medicamentos, fortaleceu as empresas brasileiras que passaram a produzir os fármacos genéricos no Brasil, muito embora os princípios ativos por serem, em sua quase totalidade, importados da Índia e da China, aumentaram o déficit na balança comercial brasileira. Hoje, $\sim 1 / 4$ dos medicamentos comercializados no país são genéricos. ${ }^{25}$ Com a ascensão das classes sociais $\mathrm{C}, \mathrm{D}$ e E, e o aumento da expectativa de vida da população brasileira, as vendas de medicamentos genéricos tendem a aumentar. Além do aumento de consumo de genéricos, alguns dos fármacos mais vendidos no mundo vêm tendo suas patentes expiradas. Isso gera mais oportunidades para o empresariado farmacêutico nacional. A Tabela 1 ilustra alguns medicamentos de marca que tiveram suas patentes vencidas em 2012, distribuídos entre várias classes terapêuticas (e.g. cardiovasculares, sistema nervoso central, úlcera péptica), de diversas empresas, indicando o volume de vendas mundiais em período próximo ao vencimento de suas patentes (2010).

Tabela 1. Fármacos que observaram o vencimento do período de proteção patentária em 2012, nos principais mercados, com nomes fantasia e genérico e total de vendas mundiais em 2010

\begin{tabular}{cccc}
\hline Marca $^{\mathrm{R}}$ & Nome & Empresa & $\begin{array}{c}\text { Vendas 2010 (US\$ } \\
1000)\end{array}$ \\
\hline Seroquel & Quetiapina & AZ & 6800 \\
Lexapro & Escitalopram & Lundbeck & 2300 \\
Provigil & Modafanila & Teva & 1100 \\
Plavix & Clopidogrel & Sanofi/BMS & 8900 \\
Singulair & Montelucaste & Merck & 5500 \\
Actos & Pioglitazona & Takeda & 4300 \\
Diovan & Valsartana & Novartis & 4100 \\
\hline
\end{tabular}

$\mathrm{AZ}=$ Astra-Zeneca; BMS = Bristol-Meyers Squibb.

O que falta para o Brasil se transformar num player na descoberta de novos fármacos tendo em vista que apenas dois fármacos foram, efetivamente, desenvolvidos no Brasil chegando às prateleiras das farmácias? Por que os princípios ativos dos genéricos continuam sendo importados da Índia e da China?

Para tentar responder a estas perguntas, foram elencados pontos críticos e pontos positivos do setor farmacêutico brasileiro, sob a ótica da academia. Certamente os empresários têm outras respostas.

\section{PONTOS CRÍTICOS}

- Não há no país laboratórios de escalonamento primário, certificados e capacitados para adaptarem as rotas de síntese de moléculas desenvolvidas nas bancadas dos laboratórios acadêmicos.

- Número reduzido de doutores em atividades de P\&D na indústria farmacêutica.

- A total dependência da importação de princípios ativos da China e da Índia para produção de genéricos.

- Formação inadequada da imensa maioria dos profissionais farmacêuticos, que não tem durante a graduação nos cursos de Farmácia o treinamento efetivo de metodologia científica de pesquisa, desejável para a sua inserção profissional na indústria farmacêutica.

- A pouca proximidade da academia com a indústria farmacêutica. ${ }^{26}$

- O empresário industrial farmacêutico brasileiro, em sua imensa maioria, não se interessa pelos medicamentos inovadores, porque o custo para o seu desenvolvimento é elevado e o retorno financeiro é de alto risco.

- A política rígida de preços de medicamentos do governo e a pouca articulação do setor público e privado.

- A frágil estrutura da pesquisa clínica e pré-clínica para a descoberta de fármacos é agravada porque no Brasil antes de se começar um estudo com participação internacional é exigida dupla aprovação ética: pela Comissão Nacional de Ética em Pesquisa, subordinada ao Conselho Nacional de Saúde, e pelos comitês locais, além da autorização da Anvisa. Ao contrário do que acontece nas nações desenvolvidas como Estados Unidos, Japão e países da União Europeia, cujo trâmite é de no máximo de 60 dias, no Brasil espera-se mais de 1 ano.

\section{PONTOS POSITIVOS}

- No Brasil há alguns poucos grupos de pesquisa com competência acadêmica estabelecida e reconhecida em síntese orgânica. A melhor prova dessa afirmação são as sínteses recentes da atorvastatina (6, Lipitor ${ }^{\mathrm{R}}$; Figura 5), importante fármaco da classe das estatinas (vide supra), por pesquisadores do Instituto de Química da UNICAMP, integrantes do INCT de Fármacos e Medicamentos (INCT-INOFAR) e do sunitinibe (7; Sutent ${ }^{\text {R }}$ Figura 5), representante dos tinibes, por pesquisadores do Instituto de Química da UFRJ, membros do mesmo INCT. ${ }^{27}$

O Lipitor ${ }^{R}$ foi o medicamento mais vendido no mundo em todos os tempos e sua patente expirou no principal mercado farmacêutico mundial em novembro de 2011, tendo como indicação o controle dos níveis de colesterol plasmático. O Sutent ${ }^{\mathrm{R}}$, ainda protegido por patente até 2021 em alguns importantes mercados farmacêuticos,

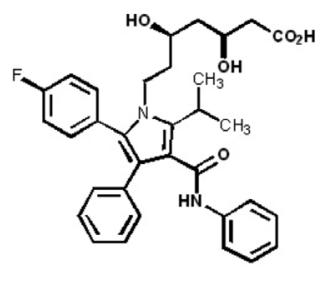

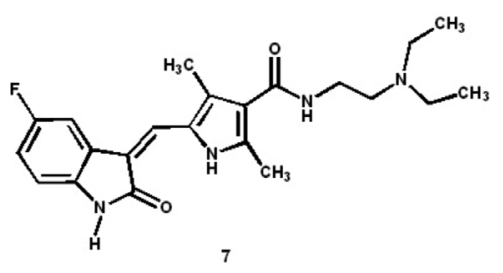

Figura 5. Estruturas dos fármacos atorvastatina (6) e sunitinibe (7) 
é usado para combater certos tipos de câncer de rim, estômago e intestino. Seu preço é elevado e o fármaco não faz parte do protocolo do Sistema Único de Saúde (SUS) brasileiro.

- O apoio financeiro do Banco Nacional de Desenvolvimento Econômico e Social (BNDES) para produção, pesquisa, desenvolvimento, inovação e fortalecimento das empresas farmacêuticas nacionais. ${ }^{28}$

- O lançamento de chamadas públicas, através de editais, como a da Biolab, o quarto maior laboratório nacional, com a FAPESP, voltada à inovação em medicamentos para a saúde humana. ${ }^{29}$

- A criação das Parcerias Público-Privadas (PPPs) para estimular as farmacoquímicas brasileiras a superarem a estagnação tecnológica atual e a aumentarem a produção de medicamentos e, consequente, redução das importações do setor farmacêutico.

- O pujante mercado varejista de medicamentos, que cresce em ritmo chinês e ocupa a sexta posição no ranking mundial.

\section{CONCLUSÕES}

Não basta só o poder de compra do governo, é necessário que as empresas farmacêuticas verticalizem a produção de fármacos e não se limitem a importação de intermediários avançados. É preciso que invistam na descoberta de fármacos inovadores.

Os cursos de graduação de Farmácia e de Química precisam adequar suas grades curriculares para formar profissionais mais qualificados para atuarem nos laboratórios de $\mathrm{P} \& \mathrm{D}$ da indústria farmacêutica.

Há muitos e bons diagnósticos do setor químico-farmacêutico. Falta, entretanto, vontade política do governo e menos burocracia, arrojo dos empresários nacionais e engajamento da academia para que o Brasil ocupe uma posição de destaque no cenário farmacêutico internacional.

\section{AGRADECIMENTOS}

Os autores agradecem ao CNPq e FAPERJ pelo apoio financeiro ao INCT-INOFAR (Proc. CNPq 573.564/2008-6 / FAPERJ E-1526/170.020/2008) e por bolsas de produtividade do CNPq e de Cientista do Nosso Estado da FAPERJ, respectivamente.

\section{REFERÊNCIAS}

1. De Andrade, J. B.; Cadore, S.; Vieira, P. C.; Zucco, C.; Pinto, A. C.; Quim. Nova 2003, 26, 445.

2. Pinto, A. C.; Zucco, C.; Galembeck, F.; de Andrade, J. B.; Vieira, P. C.; Quim. Nova 2012, 35, 2092.
3. Rosenberg, G.; Fonseca, M. G. D.; D’Avila, L. A.; Econ. Soc. 2010, 19, 107.

4. http://www.guiadafarmacia.com.br/edicao-246-tendencia/corrida-pelolucro acessada em Outubro 2013.

5. Wong, G. H. W.; Nature Biotechnology 2004, 22, 1481.

6. Benchimol, J. L. Em Louis Pasteur \& Oswaldo Cruz: Inovação e tradição em saúde; Lima, N. T.; Marchand, M-H.; eds, Editora FIOCRUZ/Banco BNP Paribas Brasil S.A.: Rio de Janeiro, 2005, cap.1.

7. Calixto, J. B.; Siqueira Jr., J. M.; Gaz. Med. Bahia 2008, 78 (suplemento), 98.

8. Cytrynowicz, M. M.; Origens e trajetórias da indústria farmacêutica no Brasil; Narrativa Um, 2007, 192 p.

9. Medicamentos inovadores são aqueles que apresentam maior eficácia em relação aos medicamentos existentes para a mesma indicação terapêutica; mesma eficácia com diminuição significativa dos efeitos adversos; ou a mesma eficácia com redução significativa do custo global (custos indiretos e diretos incluídos) do tratamento.

10. Mullard A.; Nat. Rev. Drug Discovery 2013, 11, 87; Mullard A.; Nat. Rev. Drug Discovery 2010, 9, 82; Mullard A.; Nat. Rev. Drug Discovery 2011, 10,6 .

11. Khanna, I.; Drug Discov. Today 2012, 17, 1088.

12. Paul, S. M.; Nat. Rev. Drug Discovery 2010, 9, 203.

13. Jorgensen, W. L.; Angew. Chem. Int. Ed. 2012, 51, 11680.

14. Frye, S.; Nat. Rev. Drug Discovery 2011, 10, 409.

15. Tralau-Stewart, C. J.; Drug Discov. Today 2009, 14, 95.

16. Wyatt, P. G.; Future Med. Chem. 2009, 1, 1013.

17. http://www.nobelprize.org, acessada em Outubro 2013.

18. Anderson, P. S.; Annual Reports in Medicinal Chemistry 2012, 47, 3.

19. Sanderson, K.; Nat. Rev. Drug Discovery. 2013, 12, 649.

20. Aggarwal, S.; Nat. Rev. Drug Discovery 2010, 9, 427.

21. Cohen, P.; Nat. Rev. Drug Discovery 2002, 1, 309.

22. http://www.gazetadopovo.com.br/economia/conteudo. phtml?id=1378002\&tit, acessada em Outubro 2013.

23. ht tp://www.istoedinheiro.com.br/noticias / 65181 FUSAO\%2BDE\%2BIGUAIS, acessada em Outubro 2013.

24. http://www.farmacia.ufg.br/pages/33284, acessada em Outubro 2013.

25. Barreiro, E. J. L.; Pinto, A. C.; J. Braz. Chem. Soc. 2010, 21, 775.

26. Rocha, M. M.; Lima, G. B. A.; Lameira, V. J.; Quelhas, O.L. G.; J. Technol. Manag. Innov. 2012, 7, 148.

27. Portal dos Fármacos; http://www.portaldosfarmacos.ccs.ufrj.br/ acessada em Outubro 2013.

28. Palmeira Filho, P. L.; Pieroni, J. P.; Antunes, A.; Bomtempo, J.V.; Revista do BNDES 2012, 37, 67.

29. Chamada de propostas de pesquisa FAPESP-BIOLAB - 2012 no âmbito do acordo de cooperação científica entre FAPESP e BIOLAB para apoio à pesquisa. http://www.fapesp.br/7127.phtml 